\title{
Yrast studies of ${ }^{80,82} \mathrm{Se}$ using deep-inelastic reactions
}

G. A. Jones, ${ }^{1}$ P. H. Regan, ${ }^{1}$ Zs. Podolyák, ${ }^{1}$ N. Yoshinaga, ${ }^{2}$ K. Higashiyama,${ }^{3}$ G. de Angelis,${ }^{4}$ Y. H. Zhang, ${ }^{5}$ A. Gadea, ${ }^{4}$ C. A. Ur, ${ }^{6}$ M. Axiotis, ${ }^{4}$ D. Bazzacco, ${ }^{6}$ D. Bucurescu, ${ }^{7}$ E. Farnea, ${ }^{6}$ W. Gelletly, ${ }^{1}$ M. Ionescu-Bujor, ${ }^{7}$ A. Iordachescu, ${ }^{7}$ Th. Kröll, ${ }^{4}$ S. D. Langdown, ${ }^{1}$ S. Lenzi, ${ }^{6}$ S. Lunardi, ${ }^{6}$ N. Marginean, ${ }^{4}$ T. Martinez, ${ }^{4}$ N. H. Medina, ${ }^{8}$ R. Menegazzo, ${ }^{6}$ D. R. Napoli, ${ }^{4}$ B. Quintana, ${ }^{9}$ B. Rubio, ${ }^{10}$ C. Rusu, ${ }^{11}$ R. Schwengner, ${ }^{12}$ D. Tonev ${ }^{4}$ J. J. Valiente Dobón, ${ }^{1,4}$ and W. von Oertzen ${ }^{13}$ ${ }^{1}$ Department of Physics, University of Surrey, Guildford GU2 7XH, United Kingdom

${ }^{2}$ Department of Physics, Saitama University, Saitama City 338-8570, Japan

${ }^{3}$ Department of Physics, Chiba Institute of Technology, Narashino, Chiba 275-0023, Japan

${ }^{4}$ Istituto Nazionale di Fisica Nucleare (INFN), Laboratori Nazionali di Legnaro, Italy

${ }^{5}$ Institute of Modern Physics, CAS, Lanzhou, People's Republic of China

${ }^{6}$ INFN Sezione di Padova, Italy

${ }^{7}$ National Institute for Physics and Nuclear Engineering, Bucharest, Romania

${ }^{8}$ Instituto de Fisica, Universidade de Sao Paulo, Sao Paulo, Brazil

${ }^{9}$ Grupo de Fisica Nuclear, Universidade de Salamanca, Spain

${ }^{10}$ Instituto de Fisica Corpuscular, Valencia, Spain

${ }^{11}$ INFN Laboratori Nazionali di Legnaro, Legnaro, Italy

${ }^{12}$ Institut für Kern und Hadronenphysik, Forschungszentrum Rossendorf, Dresden, Germany

${ }^{13}$ Freie Universität Berlin, Fachbereich Physik, Berlin, Germany

(Received 18 May 2007; published 26 November 2007; publisher error corrected 10 December 2007)

\begin{abstract}
We report the results of an experiment in which we studied the near-yrast states in selenium isotopes approaching $N=50$ following their population in multinucleon transfer reactions between a ${ }^{82} \mathrm{Se}$ beam and a ${ }^{192} \mathrm{Os}$ target. The level schemes for ${ }^{80,82} \mathrm{Se}$ derived from the current work are compared with restricted-basis shell-model calculations and pair-truncated shell-model calculations. These provide a good description of the yrast sequences in these nuclei using a basis space limited to excitations in the $v\left(p_{\frac{3}{2}}, p_{\frac{1}{2}}, g_{\frac{9}{2}}\right)$ and $\pi\left(f_{\frac{5}{2}}, p_{\frac{3}{2}}, p_{\frac{1}{2}}\right)$ orbitals.
\end{abstract}

DOI: 10.1103/PhysRevC.76.054317

PACS number(s): 21.10.-k, 21.60.Cs, 25.70.Hi, 27.50.+e

The study of neutron-rich nuclei is of significant current interest. Of particular focus is the question of the robustness of magic numbers at $N, Z=28,40$, and 50, and the appearance of subshell closures at nucleon numbers 32 and 34 as a function of neutron/proton number. Single-particle monopole energy shifts between specific orbitals can result in a rearrangement of the traditional ordering of subshells associated with nearspherical nuclei [1,2], which in turn results in the appearance (removal) of new (traditional) magic numbers. Recent reports following initial studies with projectile fragmentation [3-5] and deep-inelastic reactions [6-9] have suggested a possible subshell closure for neutron number $N=32$ (and perhaps $N=34$ ), associated with a large single-particle gap between the neutron $p_{\frac{3}{2}}$ and $p_{\frac{1}{2}}$ orbitals. It has been proposed that a weakening of the proton-neutron $\pi 1 f_{\frac{7}{2}} \rightarrow \nu 1 f_{\frac{5}{2}}$ interaction and a significant $\nu 2 p_{\frac{3}{2}} \rightarrow \nu 2 p_{\frac{1}{2}}$ spin-orbit splitting result in the appearance of an $N=32$ shell closure by lowering the $p$-shell neutron orbitals in energy with respect to the $f_{\frac{5}{2}}$ states. The states are inverted compared to the traditional near-stable ordering in which it is expected that the $f_{\frac{5}{2}}$ lies below the $p_{\frac{3}{2}}$ and $p_{\frac{1}{2}}$, respectively.

Monopole-energy shifts can be inferred from direct measurement of single-particle energies in odd- $A$ nuclei or, alternatively, by identifying configuration-specific decays in the spectral sequences at higher spins [10]. This short article is intended to investigate the proton energy levels above the $Z=28$ shell closure for $N \sim 50$.
The nuclei of interest were populated following heavy-ion, multinucleon transfer reactions with a thick target $[8,9,11,12]$. The beam of ${ }^{82} \mathrm{Se}$ ions of energy $460 \mathrm{MeV}$ was provided by the tandem XTU and superconducting LINAC ALPI at the Laboratori Nazionali di Legnaro, Italy. The ions were incident on an isotopically enriched $(97.8 \%){ }^{192}$ Os target of thickness $50 \mathrm{mg} / \mathrm{cm}^{2}$ backed with a $0.2 \mathrm{~mm}$ layer of Ta, thick enough to stop all the reaction products. Reaction $\gamma$ rays were measured using the GASP spectrometer [13] consisting of 40 Compton-suppressed, coaxial, hyperpure germanium detectors and an 80 element inner BGO ball for $\gamma$-ray multiplicity and calorimetry measurements. Events consisting of at least 3 Compton-suppressed germaniums and 2 BGO elements within an event time of approximately $1 \mu \mathrm{s}$ were written to tape for off-line analysis. The on-target beam current during the experiment was approximately $2 \mathrm{pnA}$, which resulted in a typical master-trigger rate of $4 \mathrm{kHz}$ over the course of the 6 day experiment. Further details and results of this experiment are presented in Refs. [11,12,14,15]. The data were sorted into symmetric $\gamma$-ray energy coincidence cubes and analyzed using standard software packages described in Ref. [16]. Examples of double-gated $\gamma$-ray triples spectra from the cube analysis are shown in Fig. 1. The $\gamma$-ray information on the osmium isotopes has been reported elsewhere [11], but we note again here the high-spin population on the near-target nuclei in the current work. Discrete states with angular momenta of $20 \hbar$ and higher were observed in even- $A{ }^{186 \rightarrow 194}$ Os, highlighting the effectiveness of multinucleon transfer reactions for the population of near-yrast states in near-stable nuclei. 


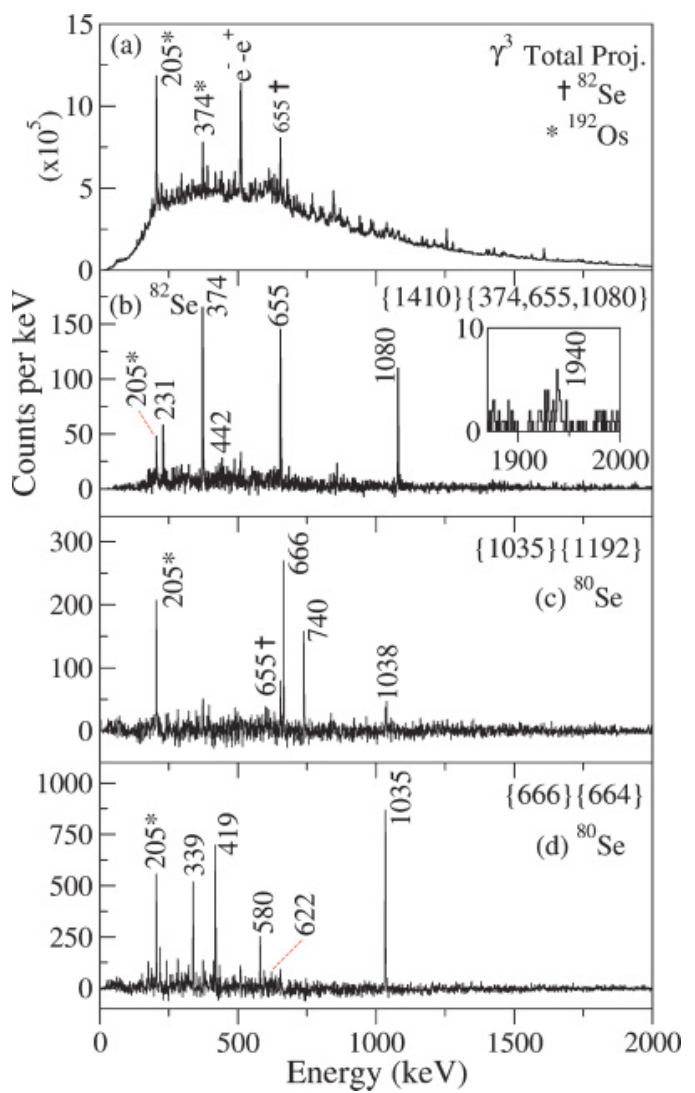

FIG. 1. (a) The total projection of $\gamma$-ray triples. Spectra in parts (b) $\rightarrow$ (d) illustrate double-gated $\gamma$-ray gates on transitions (shown in parentheses) in ${ }^{80,82} \mathrm{Se}$ from the current work.

The decay schemes associated with ${ }^{80,82}$ Se deduced from the current work are shown in Fig. 2. States up to spin $8 \hbar$ have also been reported previously in the $N=48$ isotone ${ }^{82} \mathrm{Se}$, following deep-inelastic reactions $[17,18]$, with a nanosecond isomer associated with the $\left(\nu g_{\frac{9}{2}}\right)^{-2}, I^{\pi}=8^{+}$seniority configuration at $E_{x}=3519 \mathrm{keV}$. Such two-neutron-hole seniority isomers are a standard feature throughout the $N=48$ isotonic chain [17,18]. Three $\gamma$ rays of energy 1940, 231, and $442 \mathrm{keV}$ were observed to decay from states above the $E_{x}=$ $3519 \mathrm{keV}$ level in ${ }^{82} \mathrm{Se}$. Further transitions of energies 1038, $664,622,580,419$, and $339 \mathrm{keV}$ have been observed in ${ }^{80} \mathrm{Se}$. The ordering of transitions identified in ${ }^{80,82} \mathrm{Se}$ was inferred from $\gamma$-ray intensity measurements. The thickness of the arrows in Fig. 2 indicates $\gamma$ ray intensities measured from double-gated spectra. Notably, the $1940 \mathrm{keV} \gamma$ ray feeds directly to the yrast $I^{\pi}=8^{+}$state.

Where possible, spin and parity assignments were made by comparison with previous studies of these nuclides using Coulomb excitation, $\beta^{-}$decay, and inelastic neutron scattering $[19,20]$. The statistics in the current work precluded the use of $\gamma-\gamma$ correlation analysis for further multipole assignments; however the prompt timing conditions used in the off-line analysis limit likely electromagnetic decays to $E 1, M 1, E 2$, and possibly $M 2$ character. The current data are consistent with previously reported studies of ${ }^{80,82} \mathrm{Se}$ performed using

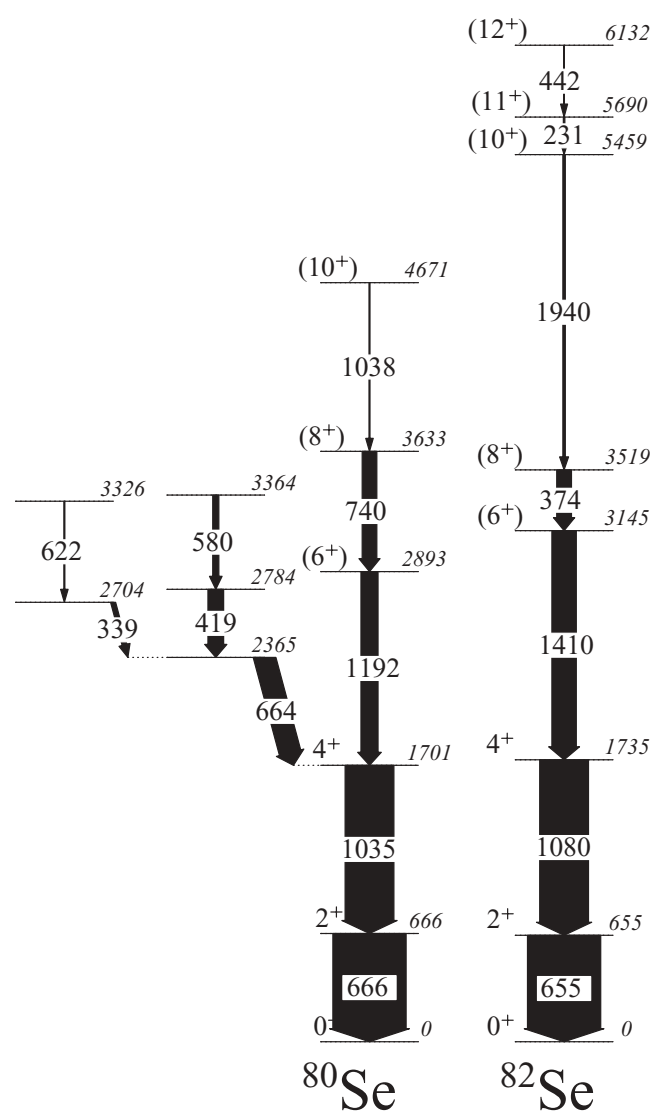

FIG. 2. Partial level schemes deduced for ${ }^{80,82} \mathrm{Se}$ in the current work. The thickness of an arrow represents the measured $\gamma$-ray intensity.

the EUROBALL array following population in fusion-fission reactions [21].

Whilst definite spin and parity assignments were not possible for states in the current work, comparison with both shell-model calculations (see below) and other $N=$ 48 isotones $[17,18,22,23]$ strongly suggests that the $1940 \mathrm{keV}$ transition represents the yrast $10^{+} \rightarrow 8^{+}$decay in ${ }^{82} \mathrm{Se}$.

Shell-model calculations were performed for ${ }^{80,82} \mathrm{Se}$ assuming a simple valence space made up of neutrons occupying $p_{\frac{3}{2}}, p_{\frac{1}{2}}$, and $g_{\frac{9}{2}}$ orbitals, while the proton valence space was restricted to the $p_{\frac{3}{2}}, p_{\frac{1}{2}}$, and $f_{\frac{5}{2}}$ levels. Here valence neutrons (protons) were treated as holes (particles) with the ${ }_{28}^{78} \mathrm{Ni}_{50}$ as closed core. The adopted single-particle energies are listed in Table I.

The effective shell-model Hamiltonian includes the monopole and quadrupole pairing plus quadrupole-quadrupole

TABLE I. Adopted single-particle energies for neutron holes and proton particles (in $\mathrm{MeV}$ ).

\begin{tabular}{lcccc}
\hline \hline$j$ & $g_{\frac{9}{2}}$ & $f_{\frac{5}{2}}$ & $p_{\frac{1}{2}}$ & $p_{\frac{3}{2}}$ \\
\hline$\varepsilon_{v}$ & 0.00 & - & 0.59 & 1.09 \\
$\varepsilon_{\pi}$ & - & 0.00 & 1.11 & 0.77 \\
\hline \hline
\end{tabular}




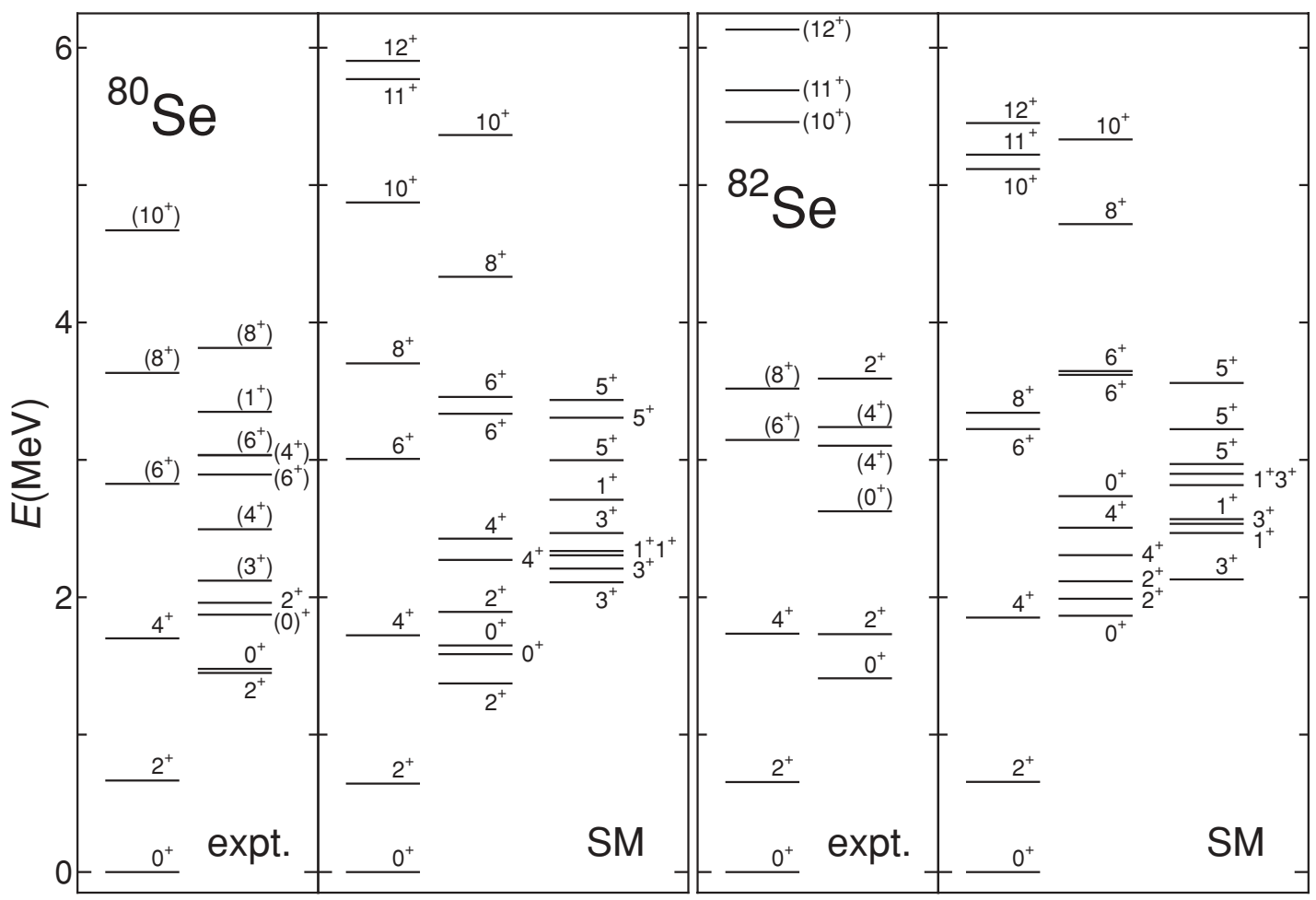

FIG. 3. Comparison between experimental levels (expt.) with shell-model results (SM) for ${ }^{80}$ Se and ${ }^{82}$ Se. Empirical state energies are taken from the current work and Refs. [19,21].

interaction and is written as

$$
\begin{aligned}
H= & \sum_{j m \tau} \varepsilon_{j \tau} c_{j m \tau}^{\dagger} c_{j m \tau}-\sum_{\tau}\left[G_{0 \tau} P_{\tau}^{\dagger(0)} P_{\tau}^{(0)}+G_{2 \tau} P_{\tau}^{\dagger(2)} \cdot \tilde{P}_{\tau}^{(2)}\right. \\
& \left.+\kappa_{\tau}: Q_{\tau} \cdot Q_{\tau}:\right]-\kappa_{\nu \pi} Q_{\nu} \cdot Q_{\pi},
\end{aligned}
$$

where $\tau(\tau=v$ or $\pi)$ stands for neutron or proton and $\varepsilon_{j \tau}, G_{0 \tau}, G_{2 \tau}, \kappa_{\tau}$, and $\kappa_{\nu \pi}$ are single-particle energies and strengths of the monopole-pairing interaction, quadrupolepairing interaction, and quadrupole-quadrupole interactions among like nucleons and unlike nucleons, respectively. The strengths of the two-body interactions for protons are fixed to reproduce the experimental energy levels of the singly closed-shell nucleus ${ }^{84} \mathrm{Se}$. The strengths of the two-body interactions for neutrons and that between neutrons and protons are adjusted to reproduce the excitation energies of the low-lying states for ${ }^{80,82} \mathrm{Se}$ and neighboring even-even nuclei. The detailed framework of the model is presented in Ref. [24]. The adopted strengths of the interactions are as follows (in $\mathrm{MeV}$ ): $G_{0 v}=0.200, G_{2 v}=0.070, \kappa_{v}=0.240, G_{0 \pi}=$ $0.260, G_{2 \pi}=0.006, \kappa_{\pi}=0.090, \kappa_{\nu \pi}=-0.200$.

In Fig. 3 the predicted energy levels are compared with the experimental data determined for the nuclei in the current work. In general, the shell-model calculations reproduce the experimental levels rather well. The shell model predicts that high spin yrast states $(I \geqslant 10)$ appear high in excitation energy $(>4 \mathrm{MeV})$. The large energy gap predicted between the yrast $10^{+}$and $8^{+}$levels in ${ }^{82} \mathrm{Se}$ can be understood simply by the increase in valence space required to allow states of this high angular momentum. It should also be noted that the shell model reproduces the energy gap between the first $6^{+}$and $8^{+}$states in
${ }^{82} \mathrm{Se}$. For a better reproduction of the $8_{1}^{+}$state, a hexadecapole pairing interaction might be necessary.

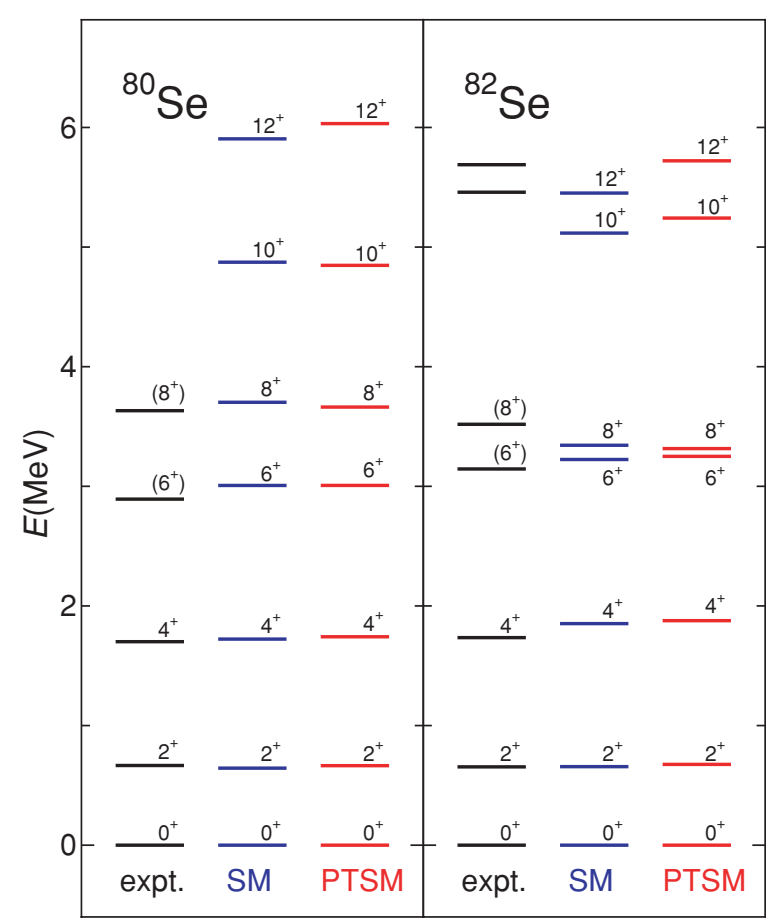

FIG. 4. (Color online) Comparison between experimental levels (expt.), shell-model results (SM), and PTSM results for ${ }^{80} \mathrm{Se}$ and ${ }^{82} \mathrm{Se}$. 


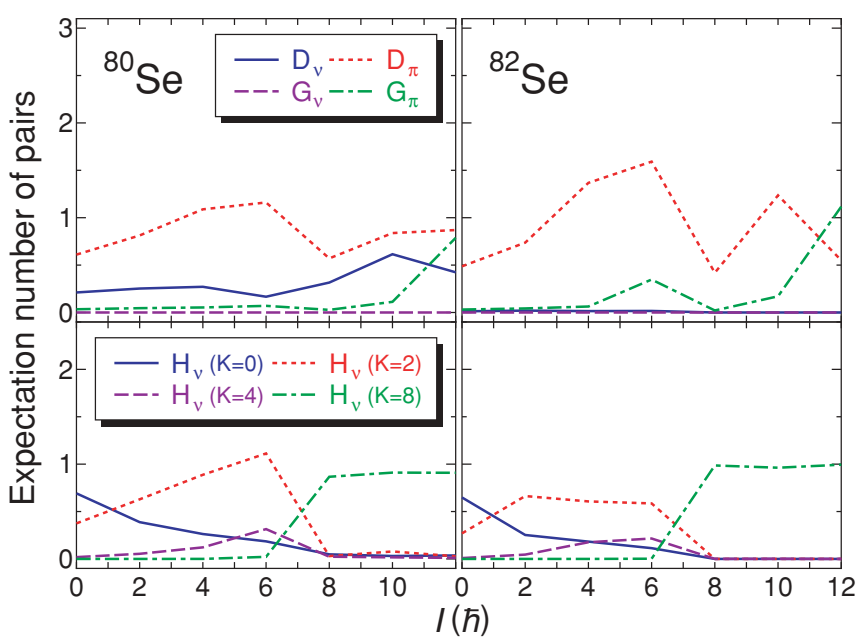

FIG. 5. (Color online) Expectation numbers of pairs in the PTSM wave functions for ${ }^{80} \mathrm{Se}$ and ${ }^{82} \mathrm{Se}$.

To better understand the structure of the low-lying states, we carried out a pair-truncated shell-model (PTSM) calculation [25] using the same effective interactions as those used in the shell-model calculations. The basis wave function is written as

$$
|\Phi(I)\rangle=\left[\left|S_{v}^{\bar{n}_{s}} D_{v}^{\bar{n}_{d}} G_{v}^{\bar{n}_{g}} H_{v}^{\bar{n}_{h}}\right\rangle \otimes\left|S_{\pi}^{n_{s}} D_{\pi}^{n_{d}} G_{\pi}^{n_{g}}\right\rangle\right]^{(I)},
$$

where $S, D$, and $G$ are the collective pairs with angular momenta 0,2 , and 4 , respectively. The $H_{\nu}$ is the noncollective pair, which is made of two neutrons in the $g_{\frac{9}{2}}$ orbital coupled to angular momentum $K(K=0,2,4,6,8)$. Except for the $K=0$ pair, twice the expectation number of $H_{\nu}$ pairs gives the seniority in the $g_{\frac{9}{2}}$ orbital.

In Fig. 4 we show the PTSM results for ${ }^{80} \mathrm{Se}$ and ${ }^{82} \mathrm{Se}$ in comparison with the experimental data and shell-model calculations. It can be seen from the figure that the model space spanned by the PTSM is adequate to reproduce the shell-model results for both nuclei. The agreement up to spin $8^{+}$is very good, as one might expect for a structure dominated by the neutron $\left(g_{\frac{9}{2}}\right)^{-2}$ seniority configurations coupled with the proton quadrupole collective excitations. The energy gap between the $I^{\pi}=6_{1}^{+}$and $8_{1}^{+}$states is explained by the "backbending phenomena." Two neutrons in the $g_{\frac{9}{2}}$ orbital align to have the maximum angular momentum 8 when the total spin changes from 6 to 8 . Of particular extra interest is the reproduction of the large energy gap between the $I^{\pi}=8_{1}^{+}$ and $10_{1}^{+}$states in ${ }^{82} \mathrm{Se}$.

In Fig. 5 the expectation numbers of various kinds of pairs in the PTSM wave functions for ${ }^{80} \mathrm{Se}$ and ${ }^{82} \mathrm{Se}$ are shown as a
TABLE II. The expectation numbers of the $S, D, G$, and $H$ pairs calculated in the PTSM for ${ }^{82} \mathrm{Se}$.

\begin{tabular}{ccccc}
\hline \hline States & $H_{v}(K=8)$ & $S_{\pi}$ & $D_{\pi}$ & $G_{\pi}$ \\
\hline $6_{1}^{+}$ & 0.00 & 1.06 & 1.59 & 0.35 \\
$8_{1}^{+}$ & 0.99 & 2.56 & 0.42 & 0.02 \\
$8_{2}^{+}$ & 0.83 & 1.52 & 1.35 & 0.13 \\
$10_{1}^{+}$ & 0.96 & 1.60 & 1.24 & 0.17 \\
$10_{2}^{+}$ & 0.94 & 0.84 & 1.34 & 0.82 \\
\hline \hline
\end{tabular}

function of spin $I$. The large energy gap between the $I^{\pi}=8_{1}^{+}$ and $10_{1}^{+}$states in ${ }^{82} \mathrm{Se}$ is mainly associated with having to allow excitation of two protons from, e.g., $f_{\frac{5}{2}}$ to $p_{\frac{3}{2}}$, which is indicated by an increase in the number of $D_{\pi}$ pairs for the $I^{\pi}=10_{1}^{+}$state compared to the $8_{1}^{+}$level in ${ }^{82} \mathrm{Se}$. We note specifically that the number of $H_{v}(K=8)$ pairs increases from zero to one from spin $6 \rightarrow 8$. This indicates that the two neutrons in the $g_{\frac{9}{2}}$ orbital align to have maximum angular momentum 8.

In Table II we show the expectation numbers of the $S, D, G$, and $H$ pairs calculated in the PTSM for ${ }^{82} \mathrm{Se}$. For the $8_{1}^{+}$state, the alignment of neutrons in the orbital $g_{\frac{9}{2}}$ dominates to give the configuration $\left(g_{9}\right)^{-2}$. This state is predicted to have very small collective excitations, evident from the calculated expectation number of $D_{\pi}$ pairs, which reduces from $1.59\left(6_{1}^{+}\right.$state $)$ to $0.42\left(8_{1}^{+}\right.$state). It is more energetically favorable for the two neutrons to align to spin 8 , rather than forming the same spin $(8 \hbar)$ by a quadrupole excitation. For the $8_{2}^{+}$state, it is seen that the contribution of the collective excitation is larger. The wave function of the $8_{1}^{+}$state and that of the $10_{1}^{+}$state are very similar except that the latter includes a quadrupole excitation. This partly explains the large energy gap between these two states.

In summary, $\gamma$-ray spectroscopy of near-yrast states in $N \sim 50$ selenium isotopes were studied using deep-inelastic reactions. The level schemes deduced from this work were compared with restricted-basis shell-model calculations and pair-truncated shell-model calculations. States in ${ }^{80,82} \mathrm{Se}$ were interpreted in terms of the alignment of $g_{\frac{9}{2}}$ neutrons and proton excitations from the $f_{\frac{5}{2}}$ subshell to the $p_{\frac{3}{2}}$ or $p_{\frac{1}{2}}$ orbital.

\section{ACKNOWLEDGMENTS}

This work is supported by the Engineering and Physical Sciences Research Council (UK), EU Contracts ERBFMGECT 980110 and HPRI-CT-1999-00083, and a Marie Curie grant of the European Community Program IHP under Contract HPMF-CT-2002-02018.
[1] B. A. Brown, Prog. Part. Nucl. Phys. 47, 517 (2001).

[2] T. Otsuka, R. Fujimoto, Y. Utsuno, B. A. Brown, M. Honma, and T. Mizusaki, Phys. Rev. Lett. 87, 082502 (2001).

[3] A. Buürger and the RISING Collaboration, Phys. Lett. B622, 29 (2005).

[4] D.-C. Dinca et al., Phys. Rev. C 71, 041302(R) (2005).
[5] S. N. Liddick et al., Phys. Rev. C 70, 064303 (2004).

[6] R. Broda, J. Phys. G 32, R151 (2006).

[7] S. N. Liddick et al., Phys. Rev. Lett. 92, 072502 (2004).

[8] B. Fornal et al., Phys. Rev. C 70, 064304 (2004).

[9] R. V. F. Janssens et al., Phys. Lett. B546, 55 (2002). 
[10] A. F. Lisetskiy, B. A. Brown, M. Horoi, and H. Grawe, Phys. Rev. C 70, 044314 (2004).

[11] Zs. Podolyák et al., Int. J. Mod. Phys. E 13, 123 (2004).

[12] Y. Zhang et al., Phys. Rev. C 70, 024301 (2004).

[13] D. Bazzacco, in Proceedings of the International Conference on Nuclear Structure at High Angular Momentum, Ottawa, 1992, Vol. II, p. 376, Report No. AECL 10613.

[14] G. A. Jones et al., Acta Phys. Pol. B 36, 1323 (2005).

[15] G. A. Jones et al., J. Phys. G 31, S1891 (2005).

[16] D. C. Radford, Nucl. Instrum. Methods Phys. Res. A 361, 297 (1995).

[17] T. Ishii, M. Asai, A. Makishima, I. Hossain, P. Kleinheinz, M. Ogawa, M. Matsuda, and S. Ichikawa, Eur. Phys. J. A 13, 15 (2002).

[18] A. Makishima, M. Asai, T. Ishii, I. Hossain, M. Ogawa, S. Ichikawa, and M. Ishii, Phys. Rev. C 59, R2331 (1999).
[19] J. K. Tuli, Nucl. Data Sheets 98, 209 (2003).

[20] B. Singh, Nucl. Data Sheets 105, 223 (2005).

[21] M. G. Porquet et al., in Proceedings of the Second International Workshop on Nuclear Fission and Fission-Product Spectroscopy, Seyssins, France (1998), p. 212; AIP Conf. Proc. 447, 212 (1998).

[22] E. K. Warburton, J. W. Olness, C. J. Lister, R. W. Zurmuhle, and J. A. Becker, Phys. Rev. C 31, 1184 (1985).

[23] E. K. Warburton, J. W. Olness, C. J. Lister, J. A. Becker, and S. D. Bloom, J. Phys. G 12, 1017 (1986).

[24] K. Higashiyama, N. Yoshinaga, and K. Tanabe, Phys. Rev. C 65 , 054317 (2002).

[25] N. Yoshinaga and K. Higashiyama, Phys. Rev. C 69, 054309 (2004). 